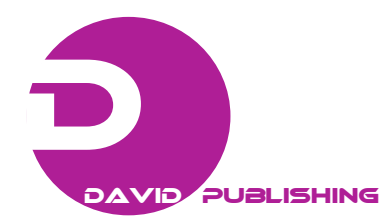

\title{
Geo-rex Rapid Analysis Assessment of Heavy Metals in the Rice, Paddy-rice and Soils
}

\author{
Akiyoshi Sawabe ${ }^{1}$, Kazuaki Nakagawa ${ }^{1}$, Ryuji Takeda ${ }^{1}$, Akira Iida ${ }^{1}$, Sadao Komemushi ${ }^{2}$, Satoshi Tamaki ${ }^{3}$ and \\ Koichiro Iwasa ${ }^{3}$ \\ 1. Faculty of Agriculture, Kinki University, 3327-204, Nakamachi, Nara-shi 631-8505, Japan \\ 2. Graduate School of Engineering, Osaka City University, 3-3-138 Sugimoto Sumiyoshi-ku, Osaka 558-8585, Japan
}

3. Sekisui Chemical Co., Ltd., 2-2 Kamichoshi-cho, Kamitoba, Minami-ku, Kyoto 601-8105, Japan

*Corresponding author's e-mail: sawabe@nara.kindai.ac.jp

\begin{abstract}
Geo-REX is a powerful tool for analyzing the heavy metals in soils and plants. Since 2005 the authors developed our new micro cartridge type compact heavy metal measuring system "Geo-REX" that did not use mercury at all to enable soil and plant analyses for on site and measured heavy metals of the whole soil or plant extracts. As for this new instrument, the detection department fits into a microcartridge entirely. After injected a $5 \mathrm{~mL}$ of sample in a cartridge, it is automatic. A new instrument can change a cartridge at every analysis. Geo-REX can measure noxious heavy metals, $\mathrm{Pb}, \mathrm{Cd}$, As, Se, Hg by exchange of a specific cartridge. Recently, the cadmium of paddy-rice and the heavy metals of the soils have become an important issue in Japan. In particular, land pollution investigation must be conducted more rapidly. In the official methods, a water dissolution test for $24 \mathrm{~h}$ is used. Quantitative analysis of heavy metals is performed by Atomic Absorption Spectrophotometry (AAS) or Inductively Coupled Plasma spectroscopy (ICP). However, it takes several days until preliminary treatment and the measurement results are available. In this study, authors compared the official method using AAS or with our new quick heavy metal measuring system based on voltammetry method. Then authors considered whether analysis of cadmium on paddy-rice investigation was possible. As for the measurement of heavy metals by microcartridge type electrochemistry analysis machine, measurement was possible in lower than 10 ppb which was Japanese reference value easily, and manipulation was simple and easy.
\end{abstract}

Key words: Geo-REX, rapid analysis, heavy metals, paddy-rice, plants, soils. 